\title{
Comparison of contrast sensitivity in myopic patients using spectacle and contact lens correction
}

\author{
SADAF QAYYUM ${ }^{1}$, ZUBAIR WAHAB ${ }^{2}$, RABIA SAEED ${ }^{3}$, ANBAR ZULFIQAR ${ }^{4}$, AYISHA SHAKEEL $^{5}$, IQRA IQBAL ${ }^{6}$ \\ ${ }^{1}$ Senior Lecturer, Pakistan Institute of Ophthalmology Rawalpindi, Pakistan \\ ${ }^{2}$ Consultant Optometrist, Al-Nafees Medical College Hospital \\ ${ }^{3}$ Lecturer, The University of Lahore \\ ${ }^{4}$ (M.phil), Pakistan Institute of Community Ophthalmology (PICO) \\ ${ }^{5}$ Associate Professor, Department of Ophthalmology, Al- Nafees Medical College Hospital \\ ${ }^{6}$ Senior Lecturer, The University of Lahore \\ Corresponding Author: Iqra lqbal, Email: Seventhsky41@yahoo.com
}

\begin{abstract}
Aim: To compare the contrast sensitivity in different categories of myopia using two different optical correction spectacles and contact lens correction.

Methods: This cross-sectional study in design was conducted from August 2018 to May 2019 at the Ophthalmology Department of Madinah Teaching Hospital Faisalabad.45 subjects corrected with spectacles lenses and contact lenses all had corrected visual acuity of $6 / 9$ or better were studied.The extent of myopia determined the three groups. All individuals were subjected to spectacles and Contact lens correction using slitlamp for anterior eye examination and for the fundus examination. The assessment of visual acuity was carried out by the Snellen vision Chart at $6 \mathrm{~m}$ distance and contrastssensitivity was tested by Pelli- Robson chart.

Results: Results showed a significant relationship between contrast sensitivity and type of optical correction. There were significant results of the independent $t$-test for spectacle and contact lenses $0.00(p<0.005)$. However, the mean contrast sensitivity was better for all the three groupswith contact lens correction as compared to spectacle lens correction.Contact lenses provide better contrast sensitivity than spectacle lenses.

Conclusion: Comparison between contact lens and spectacle correction was done and better quality contact lenses reduce optical defocus and give better results of contrast sensitivity. Results also concluded that loss of contrast sensitivity will be interpreted as early loss of retinal functions in severe myopes.

Keywords: Myopia, Contrast sensitivity, Spectacle lens, Contact lens
\end{abstract}

\section{INTRODUCTION}

Myopia or nearsightedness is a vision blinding disorder in which nearbysubstances are seen clearly, but objects which are at distant apart appear blurred. Myopia ensues if the axial length of the eyeball is too long or the cornea, the clear anterior portion of the eye, has greater curvature. Due to which, the light incomingto the eye isn't focused appropriately and distant objects appears blurred. ${ }^{1}$

The severity of myopia can be classified according to three degrees. Mild myopia (-3.00 D or Less), ModerateGmyopia (-3.00D to $-6.00 \mathrm{D})$ and High myopia (>$6.00 \mathrm{D}$ ). Risk factors for myopia include excessive time spent during near work, less exposure to Outdoor activities, advance educational level and parental history of myopia. ${ }^{2}$ The medical burden of high myopia includes pathological complications suchBas myopic macularKdegeneration, choroidal neo- vascularization, cataract, andhglaucoma. ${ }^{2}$ Visual acuity charts in different forms have been used as a standard tool for the objective and subjective determination of refractive errors. However, it is a matter of common observation that myopic patients may complain of poor vision even after objective measurements having normal $6 / 6$ visual acuity. Several studies have shown that visual acuity could be quite normal in certain types of ocular pathologies like (optic neuritis, diabetes, glaucoma, and multiple sclerosis). For this purpose, contrast sensitivity is carried out clinically ${ }^{3}$. Contrast sensitivity is the ability to

Received on 09-01-2021

Received on 19-04-4041 recognize slight deviations in luminance between regions which are not separated by specific borders is of equal importance ${ }^{4}$.

The Contrast threshold is defined as (Lmax - Lmin)/ (Lmax +Lmin), where Lmax and Lmin are the maximum and minimum luminance. Contrasttsensitivity is the inverse of the contrast threshold. It is a dimensionless quantity. Its assessment revealed signs of visual impairment, as far as changes in the appearance of fundus are concerned.Though results proposed that the deterioration of retinal structure may occur prior to ophthalmoscopic changes in the Koptic nerve head presentation, pigmentation and circulation. ${ }^{5}$

Low-grade myopia has strong $\mathrm{J}$ correlation with the refractive $\mathrm{K}$ components of the eye. For high magnitude errors axial length elongation becomes anHimportant determining factor of the refractiveeerror. Tangential stretching forces on the retina is known to induce by axial elongation in myopia.Inconsistency between the scleral shellGand choroid-retinaa is produced by the elongation of the eye. ${ }^{6}$ These changes cannmanifest themselvesFthrough an abnormal visual function e.g. decreased visual acuity, decreased Klight sensitivity, abnormal dark adaptation, visual field Kchanges and abnormal electrophysiological responses ${ }^{5}$.

With best-corrected visual acuity, myopes exhibited reduced contrast sensitivity in comparison to emmetropes. ContrastSsensitivity reduces with an increasing degree of myopia. ${ }^{7}$ Assessment of contrastssensitivity is a better predictor of individual quality of vision and visual 
performance than visual acuity. It is recommended in patients with visual impairment. It aids in the detection and evaluation of ocular pathologies . ${ }^{8}$ Pelli-Robson chart is one of the most commonly used charts for the assessment of contrast sensitivity. Its design comprises eightGrows of letters with six identical sized letters on each row. The letters are grouped into each triplet so each group of the three letters has same contrast and two sets of triplets are presented per line. The contrast decreases from $100 \%$ to $0.5 \%$ across and down the chart. The change in contrast between the successive triplets $0.15 \mathrm{log}$ units and ranges between 0.00 to 2.25 log contrast sensitivity ${ }^{9}$.

\section{MATERIAL AND METHODS}

Cross-sectional study design was used and conducted from August 2018 to May 2019 at the Department of Ophthalmology Madinah Teaching Hospital Faisalabad on both the eyes of 45 subjects of either sex aged between 20 and 40 years and informed consent was obtained from all subjects. Non- probability convenient sampling technique was used. Contrast sensitivity was measured on the 45 patients wearing both the spectacles and contact lenses. They first received contrast sensitivity testing with spectacle lenses and were examined with the soft contact lens later. All the forty five patients were regular spectacle and contact lens wearers. The subjects were divided into three groups, group 1 mild myopia (-1 D to -3 D) 15 subjects were corrected with spectacle lenses and same 15 with contact lenses. Group 2 moderate myopia (-3.25D to -6 D) including 15 subjects. 15 corrected with spectacle lenses and same 15 corrected with contact lenses. Group 3 Severe myopia (> 6D) including 15 subjects. 15 corrected with spectacle lenses and same 15 corrected with contact lenses. All the subjects free of any ocular or systemic pathology and all having 6/9 or Better visual acuity with contact lens and spectacle lens were included in the study. Astigmatism under $1.00 \mathrm{D}$ was expressed in spherical equivalent. Astigmatism greater than 1D was excluded from the study. Contrast sensitivity was measured by the Pelli-Robson contrast sensitivity chart. This test was performed binocularly and each subject was tested twice. Contrast sensitivity measurement was recorded at a distance of $1 \mathrm{~m}$. The measurements obtainedDbinocularly from each subject were articulated as a mean tstandard deviation. The analysis was done by entering the whole data into the software of SPSS20. Means of readings obtained by comparing contrast sensitivity by spectacle and contact lens correction were obtained by applying an independent sample t-test.

\section{RESULTS}

A total of 45 subjects were included in the study and total sample were divided into 3 groups (Mild, moderate and severe). Each group contains 15 subjects using spectacles and contact lens correction. The mean and standard deviation of mild group using spectacles and contact lens correction was $1.6 \pm 0.12$ and $1.88 \pm 0.78$. Similarly the mean and standard deviation of moderate group using spectacles and contact lens correction were $1.5 \pm 0.13$ and $1.85 \pm 0.10$. The mean and standard deviation of severe group using spectacles and contact lens correction were $2.7 \pm 0.16$ and $0.55 \pm 0.12$ respectively (Table 1 ).

By using independent t-test a highly significant $(p-$ 0.00) difference of contrast sensitivity was observed in different myopic groups corrected with spectacles and contact lens correction. However, there was a significant difference in the mean values of mild myopic group corrected with contact lens showed 1.88 with the standard deviation of 0.94 and mean contrast sensitivity was reduced with Spectacle lens 1.63 and a standard deviation of 0.130 . Similarly, in group 2 , there was a significant difference in the mean values of contact lens and spectacle lens. Mean contrast sensitivity was reduced with spectacle lens as compared to the contact lens. In group 3 contrast sensitivity is reduced with both optical corrections and showed a significant difference in the mean values of contrast sensitivity of both optical correction and mean contrast sensitivity was better with contact lens than spectacle lenses. It was observed the mean contrast sensitivity was better for all three groups with contact lens correction as compared to spectacles lens correction. Contact lens provides better contrast sensitivity as compared to spectacles.

Table 1: Descriptive statistics of Contrast sensitivity in 3 groups using spectacles and Contact lens

\begin{tabular}{|c|c|c|c|c|c|}
\hline \multicolumn{2}{|c|}{ Contrast sensitivity in different Myopic Group } & \multirow{3}{*}{$\frac{\mathbf{N}}{15}$} & \multirow{2}{*}{$\begin{array}{l}\text { MEAN } \\
1.6 \\
\end{array}$} & \multirow{2}{*}{$\begin{array}{l}\text { Standard Deviation } \\
0.12 \\
\end{array}$} & \multirow{3}{*}{$\begin{array}{l}\text { P- Value } \\
<0.001\end{array}$} \\
\hline Mild Group & Spectacles & & & & \\
\hline & Contact Lens & & 1.88 & 0.78 & \\
\hline \multirow[t]{2}{*}{ Moderate Group } & Spectacles & \multirow[t]{2}{*}{15} & 1.5 & 0.13 & \multirow[t]{2}{*}{$<0.001$} \\
\hline & Contact Lens & & 1.85 & 0.10 & \\
\hline \multirow[t]{2}{*}{ Severe Group } & Spectacles & \multirow[t]{2}{*}{15} & 2.7 & 0.16 & \multirow[t]{2}{*}{$<0.001$} \\
\hline & Contact Lens & & 0.55 & 0.12 & \\
\hline
\end{tabular}

\section{DISCUSSION}

Myopia causes a decrease in contrast sensitivity even after correction. A patient with $6 / 6$ visual acuity may show a decrease in contrast sensitivity might complain of visual problems .contrast sensitivity testing is recommended for the assessment of visual impairments. There are many studies that agree with this concept.

Liou and Chio in 2003 conducted research that low and moderate myopes showed no statistically significant difference with spectacle and contact lens correction ( $p$ value $<0.05$ ) and contrast sensitivity functions were also not reduced for both the groups. The patients were categorized into four myopic groups' i.e. low, medium, high and severeemyopia.contrastssensitivity was measured for all the four groups. The contrast sensitivity was reduced for group 3 myopes corrected with spectacle lens. However, no significant discrepancy was found for the contrast sensitivity functions of the myopic group with contact lens correction. Contrast sensitivity was significantly reduced for 
group 4 myopesNcorrected with both the spectacleFand contactTlens correction. ${ }^{3}$

The results of this study resembled with results of our study that there was statistical significant difference (p)value $<0.05$ was found for all the three myopic groups (mild, moderate and severe) for both the optical correction. However, there was difference in mean values of contrast sensitivity of all myopic groups corrected with spectacle lens which was less than mean values of contrast sensitivity of all the myopic groups corrected with contact lenses which conclude that contact lens enhances contrast sensitivity than spectacle lenses.

Results of the study by Collins and Carney agreed by our results in such a way that decrease in contrast sensitivity occurs in eight high myopic patients when corrected with spectacle lenses. $(p=0.0382)$ but no statistically significant difference was found with contact lens correction ( $p=0.5083$ ) between the two groups low and high myopes. Our results demonstrated that contrast sensitivity decrease with spectacle lenses in all high myopic patients as compared to contact lens correction. ${ }^{10}$

Tom- Linson, and Mann claimed that soft lens correction shows a significant increase in contrast sensitivity functions. The parallel results were found with the present study which concluded that contact lens shows better contrast sensitivity ${ }^{11}$.

Conversely, other studies show conflicting results as reported by Applegate and Massof indicate that comparing results of spectacle correction and contact lens correction indicates that contrast sensitivity was lower in subjects who wore soft contact lenses. On contrary present research revealed that by comparing both optical corrections soft contact lenses enhances contrast sensitivity ${ }^{12}$.

\section{CONCLUSION}

By comparing all the three myopic groupsmild and moderate type of myopes showed better contrast sensitivity with contact lenses had no retinal pathology. The Contact lenses reduce optical defocuse and enhance contrast sensitivity as retinal functions disturbances occur in severe myopes. Contrast sensitivity was not fully compensated by contact lenses. The Decrease in contrast sensitivity might suggest the indication for earlyHretinal function disruptionNbefore retinal pathologicalEevents occur in severe myopic patients

\section{REFERENCE}

1. Yu L, Li ZK, Gao JR, Liu JR, Xu CT. Epidemiology, genetics and treatments for myopia. Int J Ophthalmol. 2011;4(6):65869.

2. Pan CW, Ramurthy D, Saw MS. Worldwide prevalence and risk factors for myopia. OphthalPhysiol Optics.2011;32(1):316.

3. Liou.SW and Chiu CJ. Myopia and contrast sensitivity function. Current Eye Res. 2001;22(2):81-4.

4. Arden GB.The importance of measuring contrast sensitivity in cases of visual disturbance Br J Ophthalmol. 1978;62:198209.

5. Perlman I, Mayer E, Heim T, Zonis S. Retinal function in high refractive error assessed electroretinographically. $\mathrm{Br} J$ Ophthalmol. 1984;68(1):79-84.

6. Klein RM, Curtin BJ. Lacquer cracker lesions in pathologic myopia. Am J Ophthalmol. 1975;79:386-92.

7. Stoimenova BD. The effect of myopia on contrast thresholds. Invest Ophthalmol Vis Sci. 2007 May;48(5):2371-4.

8. Haymes SA, Johnston AW, Heyes AD. Relationship between vision impairment and ability to perform activities of daily living.OphthalmicPhysiolOpt. 2002;22:79-91

9. ChannaA.Do the Pelli-Robson and test chart 2000 Xpert demonstrate comparable contrast sensitivity results. $\mathrm{Br}$ IrOrthopt J. 2014;11:28-33

10. Collins J, Carney L. Visual performance in high myopia. Curr Eye Res. 1990;9:217-223.

11. Linson TA, Mann G.An analysis of the visual performance and contact lenses and spectacle correction. OphthalPhysiol Opt. 1985; 5: 53-7

12. Applegate. R. A and Massof. R. W. Changes in the contrast sensitivity function induced by contact lens wear. Am. J.Optom. Physiol. Opt. 1975;52:840-846. 